\title{
Rotated Explicit Group FDTD Method for Solving Electromagnetic Wave Propagation
}

\author{
N. M. Nusi and M. Othman, Member, IACSIT
}

\begin{abstract}
In this paper, the rotated explicit group method for solution of time domain two dimensional electromagnetic wave propagation is derived using the rotated finite difference approximation. The method is unconditionally stable and provides a significant savings in the computational time compared to the other standard methods of natural ordering.
\end{abstract}

Index Terms-Maxwell's equation, FDTD, scalar wave equation, crank-nicolson, explicit decoupled group (EDG).

\section{INTRODUCTION}

Finite Difference Time Domain (FDTD) method is one of the most commonly used numerical methods for the simulation of wave propagation. This method, known as Yee's algorithm, computes the field components by discretizing the Maxwell's curl equations both in time and space, and then solving the discretized equations in a time marching sequence by alternatively calculating the electric and magnetic fields in the computational domain [1].

Recently, a reduced scalar version of the FDTD method was developed by Aoyagi et. al [2]. In comparison with the FDTD method, the new version called the scalar wave equation finite difference time domain (WE-FDTD) requires less computation and storage. As both the FDTD and WE-FDTD methods are based on an explicit finite difference algorithm, the Courant-Friedrichs-Lewy (CFL) condition must be satisfied. A maximum time step size is limited by the minimum cell size in a computational domain. To overcome this problem, implicit methods must be employed with have no limit on the time-step size arising from the stability consideration. However, in each time step a global system of equations has to be solved of which will need more cpu time and suffer from large numerical dispersion error. Solving the problems by discretizing its computational domains in a group of points may reduce the computational time and give as good results as the conventional methods. This was proven when Evans and Abdullah skillfully developed the explicit group method according to the asymmetric Saul'yev scheme and applied the method to the solution of parabolic equations, Burger equations, diffusion equations, etc ([3]-[5]). Later Abdullah and Othman [6] developed the techniques known

Manuscript received August 1, 2012; revised September 20, 2012. This work was supported in part by the Ministry of Higher Education of Malaysia under the fundamental research grant (FRGS) Grant No. 02-01-07321FR.

N. M. Nusi is with the College of Engineering, UNITEN, and currently a post graduate student of the Institute for Mathematical Research, UPM, Malaysia (e-mail: mnoraini@uniten.edu.my).

M. Othman is with the Department of Communication Technology and Network and the associate researcher of the Institute for Mathematical Research, UPM, Malaysia (e-mail: mothman@fsktm.upm.edu.my). as explicit decoupled method (EDG) and modified explicit group (MEG) method to reduce the algorithm complexity that arises by using explicit group method on elliptic problems. All these methods are favorable in parallelism due to their explicit nature.

In this paper, we extend the concept of explicit decoupled group method for solution of two dimensional electromagnetic wave propagation. We derived the solving formula for a group of points using rotated Crank-Nicolson finite difference scheme which is unconditionally stable. Numerical simulations were carried out on Sun-Fire-v240 machine with one processor and significant savings in the computational time were achieved.

\section{STANDARD FORMULATIONS}

Consider a two-space dimensional electromagnetic problem given by the transverse magnetic (TM) waves where the field components $E_{x}, H_{x}$ and $H_{y}$ exist.

$$
\begin{aligned}
& \frac{\partial H_{x}}{\partial t}=-\frac{1}{\mu_{0}} \frac{\partial E_{z}}{\partial y} \\
& \frac{\partial H_{y}}{\partial t}=\frac{1}{\mu_{0}} \frac{\partial E_{z}}{\partial x} \\
& \frac{\partial E_{z}}{\partial t}=\frac{1}{\varepsilon_{0}}\left[\frac{\partial H_{y}}{\partial x}-\frac{\partial H_{x}}{\partial y}\right]
\end{aligned}
$$

To reduce the algorithm complexity in the TM waves formulations, the equations can be combined in a source free two-dimensional [2] as

$$
\frac{\partial^{2} u}{\partial t^{2}}=c^{2}\left[\frac{\partial^{2} u}{\partial x^{2}}+\frac{\partial^{2} u}{\partial y^{2}}\right]
$$

where $c$ is the speed of light in the domain medium and $u=E_{z}(x, y, t)$. Equation (4) forms the basics of the WE-FDTD algorithm which can be discretized in many ways. By averaging the central difference approximation on the right-hand side of equation (4) about the points $(i, j, n+1)$ and $(i, j, n-1)$, the standard difference equation known as the Crank-Nicolson method is obtained as

$$
\begin{aligned}
& \frac{u_{i, j}^{n+1}-2 u_{i, j}^{n}+u_{i, j}^{n-1}}{\Delta t^{2}} \\
& =\frac{c^{2}}{2}\left[\left\{\frac{u_{i+1, j}^{n+1}-2 u_{i, j}^{n+1}+u_{i-1, j}^{n+1}}{\Delta^{2}}+\frac{u_{i+1, j}^{n-1}-2 u_{i, j}^{n-1}+u_{i-1, j}^{n-1}}{\Delta^{2}}\right\}\right. \\
& \left.+\left\{\frac{u_{i, j+1}^{n+1}-2 u_{i, j}^{n+1}+u_{i, j-1}^{n+1}}{\Delta^{2}}+\frac{u_{i, j+1}^{n-1}-2 u_{i, j}^{n-1}+u_{i, j-1}^{n-1}}{\Delta^{2}}\right\}\right]
\end{aligned}
$$


After simplification, the equivalent equation with $r=\lambda^{2}$ and $\lambda=c \Delta t / \Delta$ is obtained as

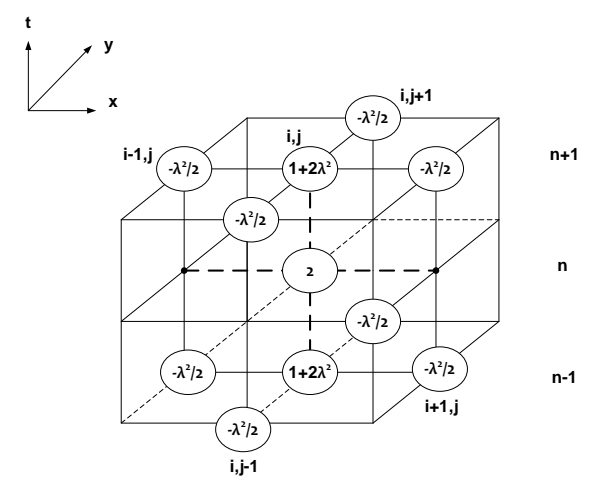

Fig. 1. Computational molecule of the crank-nicolson scheme with natural ordering

$$
\begin{aligned}
& (1+2 r) u_{i, j}^{n+1}-\frac{r}{2}\left[u_{i+1, j}^{n+1}+u_{i-1, j}^{n+1}+u_{i, j+1}^{n+1}+u_{i, j-1}^{n+1}\right] \\
& =2 u_{i, j}^{n}-(1+2 r) u_{i, j}^{n-1}+\frac{r}{2}\left[u_{i+1, j}^{n-1}+u_{i-1, j}^{n-1}+u_{i, j+1}^{n-1}+u_{i, j-1}^{n-1}\right]
\end{aligned}
$$

where $\Delta t$ is the time step and $\Delta$ is the space cell size in the $x$ and $y$ directions respectively. It has been established that this equation is unconditionally stable with principal error of order $O\left(\Delta t^{2}+\Delta^{2}\right)$. The computational molecule is shown in Fig (1).

\section{Rotated CRANK NiCOLSON Finite DifFERENCE APPROXIMATION}

Another type of finite difference that can be used to approximate (4) is the cross orientation. This can be done by rotating the $x$ and $y$ plane axis clockwise by $45^{\circ}$ with the grid spacing $\Delta \rightarrow \sqrt{ } 2 \Delta$. With the new displacement as shown in Fig (2), the rotated finite difference approximation for (4) becomes

$$
\begin{gathered}
\frac{u_{i, j}^{n+1}-2 u_{i, j}^{n}+u_{i, j}^{n-1}}{\Delta t^{2}}=\frac{c^{2}}{4}\left[\left\{\frac{u_{i+1, j+1}^{n+1}-2 u_{i, j}^{n+1}+u_{i-1, j-1}^{n+1}}{\Delta^{2}}\right.\right. \\
+\frac{\left.u_{i+1, j+1}^{n-1}-2 u_{i, j}^{n-1}+u_{i-1, j-1}^{n-1}\right\}}{\Delta^{2}} \\
\left.+\left\{\frac{u_{i-1, j+1}^{n+1}-2 u_{i, j}^{n+1}+u_{i+1, j-1}^{n+1}}{\Delta^{2}}+\frac{u_{i-1, j+1}^{n-1}-2 u_{i, j}^{n-1}+u_{i+1, j-1}^{n-1}}{\Delta^{2}}\right\}\right]
\end{gathered}
$$

Upon simplification, the following finite difference equation is obtained:

$$
\begin{gathered}
(1+r) u_{i, j}^{n+1}-\frac{r}{4}\left[u_{i+1, j+1}^{n+1}+u_{i-1, j-1}^{n+1}+u_{i-1, j+1}^{n+1}\right. \\
\left.+u_{i+1, j-1}^{n+1}\right]=2 u_{i, j}^{n}-(1+r) u_{i, j}^{n-1} \\
+\frac{r}{4}\left[u_{i+1, j+1}^{n-1}+u_{i-1, j-1}^{n-1}+u_{i-1, j+1}^{n-1}+u_{i+1, j-1}^{n-1}\right]
\end{gathered}
$$

The rotated scheme is also unconditionally stable and has a principal local truncation error of order $O\left(\Delta t^{2}+\Delta^{2}\right)$.

\section{IMPLEMENTATION OF THE EDG METHOD}

We develop the solving formula for the explicit group points using rotated finite difference scheme based on (6) for any group of four points $A(i, j), B(i+1, j+1), C(i+1, j)$ and $D(i, j+1)$ on the discretized domain at any time level $n+1$.

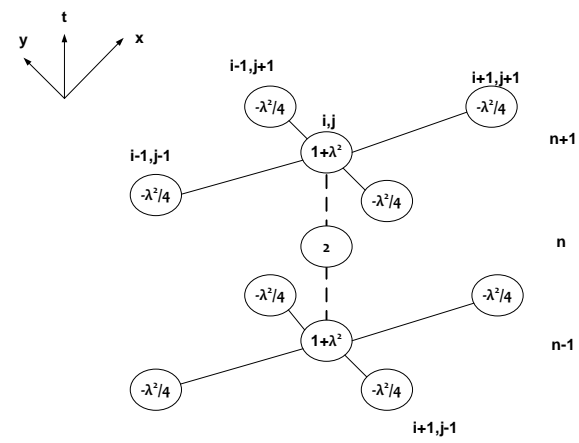

Fig. 2. Computational molecule of the rotated crank-nicolson scheme with natural ordering

Thus, will result in $4 \times 4$ implicit system matrix form as

$$
\left[\begin{array}{cccc}
\alpha & -\beta & 0 & 0 \\
-\alpha & \alpha & 0 & 0 \\
0 & 0 & \alpha & -\beta \\
0 & 0 & -\beta & \alpha
\end{array}\right]\left[\begin{array}{c}
u_{i, j} \\
u_{i+1, j+1} \\
u_{i+1, j} \\
u_{i, j+1}
\end{array}\right]^{n+1}=\left[\begin{array}{c}
b_{1} \\
b_{2} \\
b_{3} \\
b_{4}
\end{array}\right]
$$

where $\alpha=1+r, \beta=r / 4$ and

$$
\begin{gathered}
b_{1}=\beta\left[u_{i-1, j-1}^{n+1}+u_{i-1, j+1}^{n+1}+u_{i+1, j-1}^{n+1}\right]+2 u_{i, j}^{n}-\alpha u_{i, j}^{n-1} \\
+\beta\left[u_{i+1, j+1}^{n-1}+u_{i-1, j-1}^{n-1}+u_{i-1, j+1}^{n-1}+u_{i+1, j-1}^{n-1}\right] \\
b_{2}=\beta\left[u_{i+2, j+2}^{n+1}+u_{i, j+2}^{n+1}+u_{i+2, j}^{n+1}\right]-\alpha u_{i+1, j+1}^{n-1}+2 u_{i+1, j+1}^{n} \\
+\beta\left[u_{i+2, j+2}^{n-1}+u_{i, j}^{n-1}+u_{i, j+2}^{n-1}+u_{i+2, j}^{n-1}\right] \\
b_{3}=\beta\left[u_{i, j-1}^{n+1}+u_{i+2, j+1}^{n+1}+u_{i+2, j-1}^{n+1}\right]+2 u_{i+1, j}^{n}-\alpha u_{i+1, j}^{n-1} \\
+\beta\left[u_{i+2, j+1}^{n-1}+u_{i, j-1}^{n-1}+u_{i+2, j-1}^{n-1}+u_{i, j+1}^{n-1}\right] \\
b_{4}=\beta\left[u_{i+1, j+2}^{n+1}+u_{i-1, j}^{n+1}+u_{i-1, j+2}^{n+1}\right]-\alpha u_{i, j+1}^{n-1}+2 u_{i, j+1}^{n} \\
+\beta\left[u_{i+1, j+2}^{n-1}+u_{i-1, j}^{n-1}+u_{i-1, j+2}^{n-1}+u_{i+1, j}^{n-1}\right]
\end{gathered}
$$

The system leads to a decoupled system of $2 \times 2$ equations which can be written in explicit form as:

$$
\left[\begin{array}{c}
u_{i, j} \\
u_{i+1, j+1}
\end{array}\right]^{n+1}=\frac{16}{15 r^{2}+32 r+16}\left[\begin{array}{cc}
\alpha & \beta \\
\beta & \alpha
\end{array}\right]\left[\begin{array}{l}
b_{1} \\
b_{2}
\end{array}\right]
$$

and

$$
\left[\begin{array}{l}
u_{i+1, j} \\
u_{i, j+1}
\end{array}\right]^{n+1}=\frac{16}{15 r^{2}+32 r+16}\left[\begin{array}{cc}
\alpha & \beta \\
\beta & \alpha
\end{array}\right]\left[\begin{array}{l}
b_{3} \\
b_{4}
\end{array}\right]
$$

This gives the solving formula for explicit decoupled group of two points on the solution domain. The computational molecule of the explicit decoupled group system is illustrated in figure (3). From the diagram, it can be 
observed that the iterative evaluation of equation (7) involve only points of type until a convergence criteria is met. The remaining points can be evaluated directly at the required time steps using (5) which is the standard Crank-Nicolson scheme. These equations can be implemented independently, therefore the execution time can be saved by nearly as half as it iteration is only carried out on half of the solution domain. For simplicity, we consider the number of interior points $(m-$ 1) to be an even number such that each group $G_{l}, l=1,2, \ldots,((m-1) / 2)^{2}$ consists of two points.

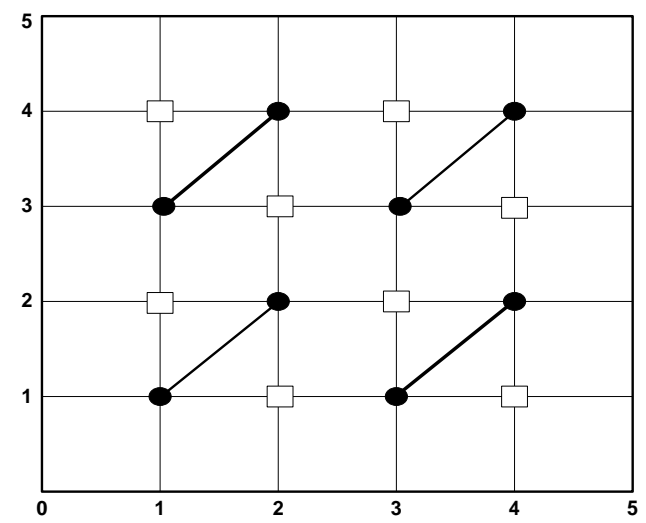

Fig. 3. The explicit decoupled group solution domain with natural ordering strategy of $m=5$

\section{NUMERICAL EXPERIMENTS AND RESULTS}

We perform the numerical simulation on (4) in a lossless medium with normalized electric permittivity and magnetic permeability, that is $\varepsilon=\mu=1$. We set the solution region as $\Omega=[0,1] \times[0,1]$ surrounded by PEC boundary conditions. The exact solution of the problem is given by

$$
E_{z}(x, y, t)=\sqrt{2} \cos (\sqrt{2} \pi t) \sin [\pi(1-x)] \sin [\pi(1-y)]
$$

The experiment was run on a Sun-Fire-v240 machine with one processor running and carried out on different grid sizes. Comparison of results for the EDG-CN are made with available results obtained from the FDTD, standard Crank-Nicolson (5) and rotated Crank-Nicolson (6) methods for various courant factors, $\lambda$.

TABLE I: COMPARISON OF NUMBER OF ITERATION (ITE), MAXIMUM ERROR (M.E), AVERAgE ABSOlute ERROR (A.A.E) AND CPU TIME OF THE METHODS WITH $\Lambda=0.25$, AFTER 10 TIME STEPS.

\begin{tabular}{llcccc}
\hline \hline$h^{-1}$ & Methods & ite & M.E & A.A.E & $\begin{array}{l}\text { CPU } \\
\text { time (s) }\end{array}$ \\
\hline 33 & FDTD & - & $7.889 \mathrm{e}-3$ & $3.497 \mathrm{e}-3$ & 0.016 \\
& CN & 5 & $8.715 \mathrm{e}-5$ & $3.759 \mathrm{e}-5$ & 0.032 \\
& Rotated-CN & 5 & $2.462 \mathrm{e}-4$ & $1.062 \mathrm{e}-4$ & 0.032 \\
& EDG-CN & 6 & $2.466 \mathrm{e}-4$ & $2.127 \mathrm{e}-4$ & 0.032 \\
\hline 65 & FDTD & - & $2.057 \mathrm{e}-3$ & $8.661 \mathrm{e}-4$ & 0.046 \\
& CN & 4 & $5.992 \mathrm{e}-6$ & $2.506 \mathrm{e}-6$ & 0.124 \\
& Rotated-CN & 4 & $1.683 \mathrm{e}-5$ & $7.036 \mathrm{e}-6$ & 0.124 \\
& EDG-CN & 5 & $1.719 \mathrm{e}-5$ & $1.417 \mathrm{e}-5$ & 0.162 \\
\hline 81 & FDTD & - & $1.326 \mathrm{e}-3$ & $5.536 \mathrm{e}-4$ & 0.154 \\
& CN & 4 & $2.501 \mathrm{e}-6$ & $1.039 \mathrm{e}-6$ & 0.426 \\
& Rotated-CN & 4 & $6.916 \mathrm{e}-6$ & $2.874 \mathrm{e}-6$ & 0.378 \\
& EDG-CN & 5 & $7.392 \mathrm{e}-6$ & $5.917 \mathrm{e}-6$ & 0.582 \\
\hline 101 & FDTD & - & $8.538 \mathrm{e}-4$ & $3.540 \mathrm{e}-4$ & 0.328 \\
& CN & 4 & $7.783 \mathrm{e}-7$ & $3.215 \mathrm{e}-7$ & 0.522 \\
& Rotated-CN & 4 & $2.743 \mathrm{e}-6$ & $1.134 \mathrm{e}-6$ & 0.528 \\
& EDG-CN & 5 & $3.135 \mathrm{e}-6$ & $2.502 \mathrm{e}-6$ & 0.611 \\
\hline \hline
\end{tabular}

The results from table (I-III) show that the EDG-CN method is relatively good as comparison to the conventional FDTD and the standard Crank-Nicolson methods in terms of accuracy. However, the rate of convergence using the EDG method is faster than the the standard Crank-Nicolson methods due to it less iteration number. Furthermore, the proposed EDG method significantly reduces the cpu time to nearly $50 \%$ and as fast as the conventional FDTD method.

TABLE II: COMPARISON OF NUMBER OF ITERATION (ITE), MAXIMUM ERROR (M.E), Average Absolute ERror (A.A.E) AND CPU Time OF THE METHODS WITH $\Lambda=1.0$, AFTER 10 TIME STEPS.

\begin{tabular}{llcccl}
\hline \hline \multirow{2}{*}{$h^{-1}$} & Methods & ite & M.E & A.A.E & $\begin{array}{l}\text { CPU } \\
\text { time (s) }\end{array}$ \\
\hline 33 & FDTD & - & $7.889 \mathrm{e}-3$ & $3.497 \mathrm{e}-3$ & 1.1 \\
& CN & 15 & $6.883 \mathrm{e}-3$ & $3.035 \mathrm{e}-4$ & 2.6 \\
& Rotated-CN & 13 & $8.744 \mathrm{e}-3$ & $3.772 \mathrm{e}-3$ & 2.3 \\
& EDG-CN & 7 & $8.738 \mathrm{e}-3$ & $7.538 \mathrm{e}-3$ & 1.2 \\
\hline 65 & FDTD & - & $3.622 \mathrm{e}-2$ & $1.284 \mathrm{e}-2$ & 4.4 \\
& CN & 14 & $6.000 \mathrm{e}-4$ & $2.509 \mathrm{e}-4$ & 7.7 \\
& Rotated-CN & 12 & $7.529 \mathrm{e}-4$ & $3.148 \mathrm{e}-4$ & 8.7 \\
& EDG-CN & 6 & $7.468 \mathrm{e}-4$ & $6.245 \mathrm{e}-4$ & 4.7 \\
\hline 81 & FDTD & - & $2.019 \mathrm{e}-2$ & $8.424 \mathrm{e}-3$ & 7.9 \\
& CN & 13 & $2.619 \mathrm{e}-4$ & $1.088 \mathrm{e}-4$ & 17.3 \\
& Rotated-CN & 11 & $3.287 \mathrm{e}-4$ & $1.366 \mathrm{e}-4$ & 16.6 \\
& EDG-CN & 6 & $3.194 \mathrm{e}-4$ & $2.654 \mathrm{e}-4$ & 8.5 \\
\hline 101 & FDTD & - & $1.323 \mathrm{e}-2$ & $5.482 \mathrm{e}-3$ & 15.1 \\
& CN & 13 & $1.194 \mathrm{e}-4$ & $4.938 \mathrm{e}-5$ & 35.1 \\
& Rotated-CN & 11 & $1.410 \mathrm{e}-4$ & $5.831 \mathrm{e}-5$ & 33.5 \\
& EDG-CN & 6 & $1.354 \mathrm{e}-4$ & $1.117 \mathrm{e}-4$ & 16.4 \\
\hline \hline
\end{tabular}

TABLE III: COMPARISON OF NUMBER OF ITERATION (ITE), MAXIMUM ERror (M.E), AVERAGE AbSOlute ERror (A.A.E) AND CPU TIME OF THE METHODS WITH $\Lambda=4.0$, AFTER 10 TIME STEPS

\begin{tabular}{llllll}
\hline \hline \multirow{2}{*}{$h^{-1}$} & Methods & ite & M.E & A.A.E & $\begin{array}{l}\text { CPU } \\
\text { time (s) }\end{array}$ \\
\hline 33 & FDTD & - & - & - & - \\
& CN & 30 & $3.244 \mathrm{e}-1$ & $1.399 \mathrm{e}-1$ & 9.2 \\
& Rotated-CN & 108 & $3.304 \mathrm{e}-1$ & $1.425 \mathrm{e}-1$ & 8.2 \\
& EDG-CN & 54 & $3.304 \mathrm{e}-1$ & $2.850 \mathrm{e}-1$ & 4.3 \\
\hline 65 & FDTD & - & - & - & - \\
& CN & 27 & $2.221 \mathrm{e}-2$ & $9.285 \mathrm{e}-3$ & 20.7 \\
& Rotated-CN & 105 & $2.258 \mathrm{e}-2$ & $9.443 \mathrm{e}-3$ & 19.2 \\
& EDG-CN & 54 & $2.247 \mathrm{e}-2$ & $1.879 \mathrm{e}-2$ & 9.4 \\
\hline 81 & FDTD & - & - & - & - \\
& CN & 132 & $2.293 \mathrm{e}-2$ & $9.529 \mathrm{e}-3$ & 27.4 \\
& Rotated-CN & 109 & $2.330 \mathrm{e}-2$ & $9.681 \mathrm{e}-3$ & 25.2 \\
& EDG-CN & 55 & $2.317 \mathrm{e}-2$ & $1.926 \mathrm{e}-2$ & 12.5 \\
\hline \multirow{2}{*}{101} & FDTD & - & - & - & - \\
& CN & 132 & $1.444 \mathrm{e}-2$ & $5.970 \mathrm{e}-3$ & 68.5 \\
& Rotated-CN & 109 & $1.464 \mathrm{e}-2$ & $6.052 \mathrm{e}-3$ & 65.6 \\
& EDG-CN & 55 & $1.449 \mathrm{e}-2$ & $1.199 \mathrm{e}-2$ & 20.3 \\
\hline \hline
\end{tabular}

\section{CONCLUSION}

We have demonstrated the implementation of the explicit decoupled group (EDG) method on two dimensional electromagnetic wave propagation based on the rotated Crank-Nicolson approximation. It is observed that the EDG method provides significant saving in the computational time compared to the other methods. In fact, the EDG method can be one of the computational tools for solving any problems related to electromagnetism as well as the FDTD method.

\section{ACKNOWLEDGMENT}

The authors would like to Lab of Computational Science and Informatics, Institute of Mathematical Research 
(INSPEM) for the computing facilities.

\section{REFERENCES}

[1] K. S. Yee, "Numerical solution of initial boundary value problem involving Maxwell's equations in isotropic media," IEEE Trans. Antennas Propagation, AP-14, pp. 302-307, 1966.

[2] P. H. Aoyagi, J.-F. Lee, and R. Mittra, "A hybrid Yee algorithm/scalar wave equation approach," IEEE Trans. Microwave Theory and Tech, vol 41, no. 19, pp. 1593-1600, 1993.

[3] D. J. Evans, "Group explicit methods for the numerical solution of partial differential equations - (Topics in Computer Mathematics)," Gordon and Breach Science Publishers, 1997.

[4] V. K. Saulyev, "Integration of equations of parabolic type equation by the method of Net," Pergamon Press, New York 1964.

[5] A. R. Abdullah, "The four point explicit decoupled group (EDG) method: a fast poisson solver," International J. Computer Mathematics, vol. 38, pp. 61-70, 1991.

[6] M. Othman and A. R. Abdullah, "An efficient four points modified explicit group poisson solver," International J. Computer Mathematics, vol. 76, pp. 203-217, 2000.

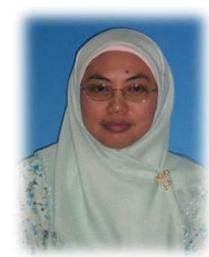

N.M. Nusi was born in Selangor, Malaysia on August 21, 1973. She obtained her Bachelor of Applied Science Degree from Universiti Sains Malaysia (USM) in 1997 and MSc from Universiti Kebangsaan Malaysia in 2002. She is now doing Ph.D degree in high performance computing in Laboratory of Computational Mathematics, Institute for Mathematical Research (INSPEM), Universiti Putra Malaysia. Her current research interests includes numerical analysis, scientific computing and computational electromagnetic.

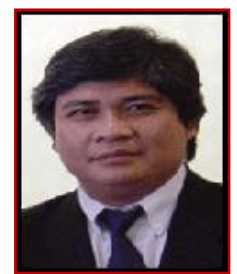

M. Othman is an Associate Professor of Computer Science in the Dept of Communication Technology and Network, Universiti Putra Malaysia (UPM), since 2001. In April 2008, he was appointed as a deputy director of InfoComm Development Centre (iDEC) at the same university. He received his $\mathrm{PhD}$ in Computer Science from the Department of Industrial Computing, Universiti Kebangsaan Malaysia with distinction (Best PhD Thesis in 2000 awarded by Sime Darby Malaysia and Malaysian Mathematical Science Society) in 1999. He is also an associate researcher and program coordinator in the areas of High Performance Computing (HPC), Computer Networks and Scientific Computing at the Laboratory of Computational Science and Informatics, INSPEM, UPM. He has expertise in several areas of computer science such as parallel and distributed algorithms, grid computing, high-speed computer network and scientific computing. 